\title{
Prognostic impact of a ground-glass opacity component in clinical stage IA non-small cell lung cancer
}

Aritoshi Hattori, MD, ${ }^{\mathrm{a}}$ Kenji Suzuki, MD, ${ }^{\mathrm{a}}$ Kazuya Takamochi, MD, ${ }^{\mathrm{a}}$ Masashi Wakabayashi, MSc, ${ }^{\mathrm{b}}$ Keiju Aokage, MD, ${ }^{\mathrm{c}}$ Hisashi Saji, MD, ${ }^{\mathrm{d}}$ and Shun-ichi Watanabe, MD, ${ }^{\mathrm{e}}$ on behalf of the Japan Clinical Oncology Group Lung Cancer Surgical Study Group

\section{ABSTRACT}

Objective: We performed a validation study to confirm the prognostic importance of the presence of a ground-glass opacity component based on data of the Japan Clinical Oncology Group study, JCOGo201, which was a prospective observational study to predict the pathological noninvasiveness of clinical stage IA lung cancer in Japan.

Methods: Among the 811 patients registered in JCOGo201, 671 were confirmed eligible by study monitoring and a central review of computed tomography. Registered c-stage IA lung cancer was less than $30 \mathrm{~mm}$ in maximum tumor size, which was classified into a with ground-glass opacity group (pure ground-glass opacity and part-solid tumor) or solid group based on the status of a ground-glass opacity component. T staging was reassigned in accordance with the 8th edition of the TNM staging system. To validate the prognostic impact, overall survival was estimated.

Results: Of the cases, $432(64 \%)$ were in the with ground-glass opacity group and $239(36 \%)$ were in the solid group with a median follow-up time of 10.1 years. The 5 -year overall survival was significantly different between the with ground-glass opacity group and solid group ( $95.1 \%$ vs $81.1 \%$ ). The 5 -year overall survival was excellent regardless of the solid component size in the with ground-glass opacity group (c-T1a or less: $97.2 \%$, c-T1b: 93.4\%, C-T1c: $91.7 \%$ ). In contrast, prognostic impact of the tumor size was definitive in the solid group (c-T1a: $87.5 \%$, c-T1b: $85.9 \%$, C-T1C: $73.7 \%$ ).

Conclusions: Favorable prognostic impact of the presence of a ground-glass opacity component was demonstrated in JCOGo201. The presence or absence of a ground-glass opacity should be considered as an important parameter in the next clinical T classification. (J Thorac Cardiovasc Surg 2021;161:1469-80)

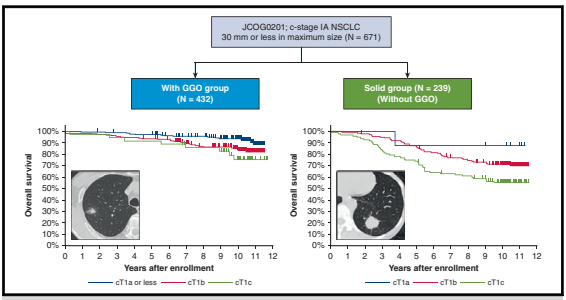

Solid tumor size differentiated survival outcome only in lung cancer without GGO.

\section{CENTRAL MESSAGE}

Favorable oncological outcome of lung cancer with a GGO component is demonstrated at a nationwide level, which is considered as a different oncological category from that of radiological solid tumor.

\section{PERSPECTIVE}

The results of a significant center validation in Japan demonstrated that the favorable prognostic impact of the presence of a GGO component was confirmed in the prospective JCOGO2O1 dataset. The presence or absence of a GGO should be strongly considered as a novel important parameter in the next clinical $\mathrm{T}$ classification.

See Commentaries on pages 1481 and 1482 .
Since the Japan Clinical Oncology Group (JCOG) study prospectively validated the radiological definition that enabled prediction of the pathological noninvasiveness of clinical stage IA lung cancer based on the findings of

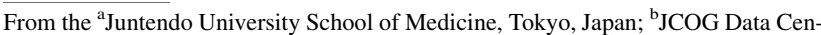
ter/Operations Office, National Cancer Center Hospital, Tokyo, Japan; ${ }^{\mathrm{c}}$ National Cancer Center Hospital East, Kashiwa, Japan; ${ }^{d}$ St Marianna University School of Medicine, Kanagawa, Japan; and ${ }^{\mathrm{N}}$ National Cancer Center Hospital, Tokyo, Japan.

This article is supported in part by Japanese National Cancer Center Research and Development Funds (23-A-16, 23-A-18, 26-A-4, 29-A-3) and Grants-in-Aid for Cancer Research (11S-2,11S-4, 14S-2, 14S-4, 17S-2, 17S-5, 20S-2, 20S-5) from the Japanese Ministry of Health, Labour, and Welfare.
}

thin-section computed tomography $(\mathrm{CT}),{ }^{1}$ many thoracic surgeons have revealed that consolidation tumor ratio (CTR) and solid component size were more prognostic than maximum tumor size for resected non-small cell

Read at the 99th Annual Meeting of The American Association for Thoracic Surgery, Toronto, Ontario, Canada, May 4-7, 2019.

Received for publication July 25, 2019; revisions received Jan 22, 2020; accepted for publication Jan 27, 2020; available ahead of print April 6, 2020.

Address for reprints: Aritoshi Hattori, MD, Department of General Thoracic Surgery, Juntendo University School of Medicine, 1-3, Hongo 3-chome, Bunkyo-ku, Tokyo 113-8431, Japan (E-mail: ahattori@juntendo.ac.jp).

$0022-5223 / \$ 36.00$

Copyright (c) 2020 by The American Association for Thoracic Surgery https://doi.org/10.1016/j.jtcvs.2020.01.107 


$$
\begin{aligned}
& \text { Abbreviations and Acronyms } \\
& \begin{array}{ll}
\text { CI } & =\text { confidence interval } \\
\text { CT } & =\text { computed tomography } \\
\text { CTR } & =\text { consolidation tumor ratio } \\
\text { GGO } & =\text { ground-glass opacity } \\
\text { HR } & =\text { hazard ratio } \\
\text { JCOG } & =\text { Japan Clinical Oncology Group } \\
\text { NSCLC } & =\text { non-small cell lung cancer } \\
\text { OS } & =\text { overall survival } \\
\text { PET } & =\text { positron emission tomography } \\
\text { RFS } & =\text { relapse-free survival }
\end{array}
\end{aligned}
$$

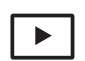

Scanning this $Q R$ code will take you to the table of contents to access supplementary information.

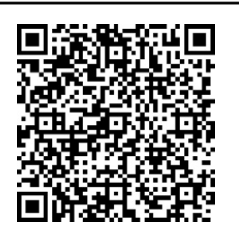

lung cancer (NCSLC). ${ }^{2-7}$ This finding is extremely important in the history of general thoracic surgery. Subsequently, the 8th edition of the TNM staging system drastically changed the staging system, with the clinical $\mathrm{T}$ category being determined according to solid component size and excluding ground-glass opacity (GGO). ${ }^{8}$ In contrast, new issues are emerging from the proposed changes concerning $\mathrm{T}$ parameters. Much of the confusion is caused by the absence of a consensus on how to make uniform the measurements of solid component size in many part-solid tumors in which solid component size is difficult or impossible to measure.

In such circumstances, we have reported a new and simple fact that the presence of a GGO denotes a great influence on the favorable prognosis of NSCLC, and the radiological solid component size is irrelevant to the survival outcome of NSCLC if the tumors show a GGO component. ${ }^{9-12}$ On the other hand, radiologically determined pure-solid lung cancers without a GGO component exhibit more malignant behavior and show several histologic types that have a poorer prognosis than do radiologically part-solid lung cancers. Thus, the prognostic impact of the solid tumor size is considered to be meaningful only in the pure-solid NSCLC. ${ }^{9-12}$ This fact is extremely important when considering future revision of the clinical $\mathrm{T}$ staging of lung cancer, provided that the clinicopathologic and oncologic outcomes are disparate between part-solid and pure-solid tumors on the basis of a GGO presence.

Despite single institution advocacy for the prognostic importance of the presence of a GGO component as a significant clinical $\mathrm{T}$ parameter, ${ }^{9-12}$ this notion has not been fully studied across institutions or at a nationwide level. To validate this fundamental and simple prognostic feature of lung cancer, we aimed to demonstrate the prognostic impact of the presence of a GGO component in clinical stage IA NSCLC based on the long-term follow-up data of JCOG0201. We hypothesized that the favorable prognosis could be confirmed regardless of the solid component size in tumors with a GGO component by the prospective JCOG0201 dataset. Moreover, the presence or absence of a GGO component would be considered an important parameter in the clinical T classification.

\section{MATERIALS AND METHODS \\ Patient Eligibility Criteria}

JCOG0201 was a prospective observational study to predict pathological noninvasiveness in clinical stage IA lung adenocarcinoma in Japan. ${ }^{1}$ The eligibility criteria were (1) a suspected or diagnosed lung cancer based on the findings from a plain X-ray or CT scan; (2) clinical stage IA in the 5th edition of the UICC TNM staging, that is, a tumor less than $30 \mathrm{~mm}$ in maximum size by thoracic enhanced CT; (3) the center of the tumor is located peripherally, that is, the outer half of the lung field on CT; (4) measurable at least in 1 dimension on thin-section CT; (5) age range 20 to 75 years, (6) no prior thoracotomy; (7) feasible for pulmonary lobectomy; and (8) obtained written informed consent. The exclusion criteria were (1) synchronous or metachronous (within 5 years) malignancy other than carcinoma in situ and (2) interstitial pneumonitis, lung fibrosis, or severe pulmonary emphysema. All patients underwent a preoperative CT scan, and hilar or mediastinal lymph nodes less than $1.0 \mathrm{~cm}$ in the shortest diameter were regarded as clinical N0. The study protocol was approved by the JCOG Clinical Trial Review Committee and by the institutional review board of each participating center. The JCOG Data Center conducted the central registration, data management, central monitoring, and statistical analysis.

Between December 2002 and May 2004, 811 patients were enrolled from 31 institutions. Of these, 140 patients were excluded in the present supplemental analysis based on the ineligibility criteria including noncancerous lesions. Finally, the prognosis of 671 patients $(82.7 \%)$ was studied on the basis of presence of a GGO component on thin-section CT scan.

\section{Radiological Evaluation of the Primary Tumor}

A contrast-enhanced CT scan was performed to evaluate the entire lung for preoperative staging. In addition, the main tumor was evaluated preoperatively to estimate the extent of GGO by thin-section helical CT scan with a 1- to 3-mm collimation. The evaluated factors on the lung window were the maximum diameters of the tumor and consolidation; the presence of a pleural tail; air bronchogram; homogeneity of the consolidation; and sharpness of the tumor margin. The consolidation component was defined as an area of increased opacification that completely obscured the underlying vascular markings. GGO was defined as an area of a slight, homogenous increase in density that did not obscure the underlying vascular markings. Furthermore, The CTR was defined as the ratio of the maximum size of consolidation to the maximum tumor size on thin-section CT scan. ${ }^{1}$ In this study, all tumors were classified into 2 groups based on the presence of a GGO component on thin-section CT scan, that is, the with GGO group $(0 \leq \mathrm{CTR}<1.0$, pure-GGO and part-solid tumor $)$ or solid group $(\mathrm{CTR}=1.0)$. Radiological semi-consolidation that had an intermediate density between solid and GGO findings was categorized in the GGO group, because they are considered to show a less-invasive nature traditionally, which is clinicopathologically similar to that of pure GGO lesions. ${ }^{2}$ Clinical $\mathrm{T}$ staging was reassigned in accordance with the 8th edition of the TNM classification. ${ }^{8}$ All tumors were defined as follows: 0 to 10 $(\mathrm{mm})=\mathrm{cT} 1 \mathrm{a}$ (or less), 11 to $20(\mathrm{~mm})=\mathrm{cT} 1 \mathrm{~b}$, and 21 to 30 $(\mathrm{mm})=\mathrm{cT} 1 \mathrm{c}$ based on the solid component size. 


\section{Central Review of Radiological Evaluation}

To ensure the final diagnosis, radiological findings based on thin-section CT were strictly reviewed by 6 experienced thoracic oncologists ( 4 thoracic surgeons and 2 radiology oncologists). This radiologic central review was indicated for patients who were preoperatively or intraoperatively diagnosed with lung cancer. CT findings were evaluated coincidentally by the six reviewers, and the final results were decided in consensus.

\section{Pathological Evaluation}

The resected specimen was sectioned at intervals of 5 to $10 \mathrm{~mm}$ throughout the whole specimen. The main tumor was sectioned into 2- to 4-mm slices, and the following pathological factors were evaluated by means of hematoxylin-eosin staining and elastic fiber staining: histologic typing; grade of differentiation; maximum tumor size and solid component size of the main tumor; pleural involvement; vascular invasion; lymphatic invasion; and intrapulmonary metastasis.

\section{Statistical Analysis}

Parts of the methods and sample size calculation have been described by Suzuki and colleagues. ${ }^{1}$ To validate the prognostic impact, overall survival (OS) was estimated. OS was defined as the duration from enrollment to death from any cause. For the patients alive, OS was censored at the last contact date. Relapse-free survival (RFS) was defined as the duration from enrollment to first recurrence or death from any cause. For the patients alive and recurrence-free, RFS was censored at the last contact date. OS and RFS were estimated by the Kaplan-Meier method. Between-group comparison of survival was performed by log-rank test. Lung cancer recurrence was estimated by cumulative recurrent incidence. Between-group comparison of lung cancer recurrence was performed by Gray's test. Univariable and multivariable analyses including all covariates were performed to adjust confounding variables for both OS and RFS. All $P$ values were 2 -sided. Hazard ratios (HRs) and $95 \%$ confidence interval (CIs) were estimated using Cox proportional hazards model. All statistical analyses were performed with SAS software release 9.4 (SAS Institute, Inc, Cary, NC) by the JCOG Data Center.

\section{RESULTS}

Of the 671 eligible cases, 407 patients showed CTR less than 1.0, and 264 patients showed CTR $=1.0$ based on the findings of thin-section CT scans. Among the patients with a tumor showing CTR $=1.0,25$ lesions $(3.7 \%)$ presented with a radiological semi-consolidation were categorized in the GGO group. Finally, $432(64 \%)$ were classified in the GGO group, and 239 (36\%) were classified in the solid group according to the result of the central review board (Figure 1).

The overall clinicopathological characteristics of the patients are summarized in Table 1 . As a result of the CT findings by the central review board, $215(50 \%)$ were categorized as c-T1a or less, $181(42 \%)$ were categorized as c-T1b, and $36(8 \%)$ were categorized as c-T1c in the with GGO group, whereas $8(3 \%)$ were categorized as c$\mathrm{T} 1 \mathrm{a}, 136(57 \%)$ as c-T1b, and $95(40 \%)$ as c-T1c in the solid group. The solid group was male predominant compared with the with GGO group $(56 \%$ vs $35 \%)$, whereas lobectomy was the main surgical procedure in both groups. With regard to the pathological characteristics, most patients in the with GGO group had adenocarcinoma of the lung $(99.3 \%)$, and several other histological types were found in the solid group. Regarding tumor differentiation, the solid group frequently showed poorly differentiated lesions. Pathological nodal involvement was found in 51 patients $(7.6 \%)$ in the entire cohort, whereas the proportion of nodal metastasis was extremely high in the solid group compared with the with GGO group (17\% vs $2 \%$, $P<.0001)$. The correlations between the frequency of nodal involvement and the T parameters are as follows: c-T1a or less $=0 \%(0 / 196)$ (with GGO: 0/190, solid: 0/6), c$\mathrm{T} 1 \mathrm{~b}=6.1 \%(19 / 314)$ (with GGO: $6 / 180=3.3 \%$, solid: $13 / 134=9.7 \% ; P=.0291)$, and $\mathrm{c}-\mathrm{T} 1 \mathrm{c}=25.0 \%$ 128) (with GGO: $4 / 34=11.8 \%$, solid: $28 / 94=29.8 \%$; $P=.0400$ ). Table 2 presents the multivariable analyses by Cox proportional hazard model, and the presence or absence of a GGO component was an independently significant prognostic factor in both OS and RFS (Table 2A, OS: HR, 1.730, 95\% CI, 1.076-2.779, $P=.0236$; Table 2B, RFS: HR, 2.169, 95\% CI, 1.388-3.991, $P=.0007)$.

The 5-year OS and 5-year RFS of the clinical stage IA NSCLC in this study were $90.1 \%$ and $84.5 \%$, respectively, with a median follow-up time of 10.1 years, which was significantly different between the with GGO and solid groups (Figure 2, A, 5-year OS: $95.1 \%$ vs $81.1 \%$, logrank test $P<.0001$, Figure 2, $B, 5$-year RFS: $93.3 \%$ vs $68.6 \%, P<.0001)$. Regarding the cause of death, $62 \%$ was not associated with lung cancer-related diseases in the with GGO tumor group. In contrast, $70 \%$ was due to lung cancer-related death in the solid tumor group. When we evaluated the survival outcomes based on solid component size, it was excellent in the with GGO group. In particular, the 5 -year OS of the with GGO group was $90 \%$ or more regardless of solid component size (Figure 3, A, 5-year OS: c-T1a or less $=97.2 \%$ [c-Tis $=97.0 \%$, $\mathrm{c}-\mathrm{T} 1 \mathrm{mi}=97.0 \%, \mathrm{c}-\mathrm{T} 1 \mathrm{a}=97.4 \%], \mathrm{c}-\mathrm{T} 1 \mathrm{~b}=93.4 \%$, $\mathrm{c}-\mathrm{T} 1 \mathrm{c}=91.7$, Figure 3, B, 5-year RFS: c-T1a or less $=96.7 \% \quad[\mathrm{c}-\mathrm{Tis}=95.5 \%, \mathrm{c}-\mathrm{T} 1 \mathrm{mi}=97.0 \%$, $\mathrm{c}-\mathrm{T} 1 \mathrm{a}=97.4 \%], \mathrm{c}-\mathrm{T} 1 \mathrm{~b}=90.6 \%, \mathrm{c}-\mathrm{T} 1 \mathrm{c}=86.1 \%)$. The difference in OS among the adjacent $\mathrm{T}$ staging was not significant in the with GGO group (c-T1a with GGO vs c-T1b with GGO: HR, 1.959, 95\% CI, 0.951-4.036, c-T1b with GGO vs c-T1c with GGO: HR, 1.438, 95\% CI, 0.6563.156). On the other hand, the prognostic impact of tumor size was definitive in the solid group. Both the 5-year OS and 5-year RFS split almost fairly among the different solid component sizes, where difference in survival was especially significant between $\mathrm{c}-\mathrm{T} 1 \mathrm{~b}$ and $\mathrm{c}-\mathrm{T} 1 \mathrm{c}$ in the solid group (c-T1b solid vs c-T1c solid: HR, 1.791, 95\% CI, 1.158-2.770) (Figure 4, A, 5-year OS: c-T1a $=87.5 \%$, 


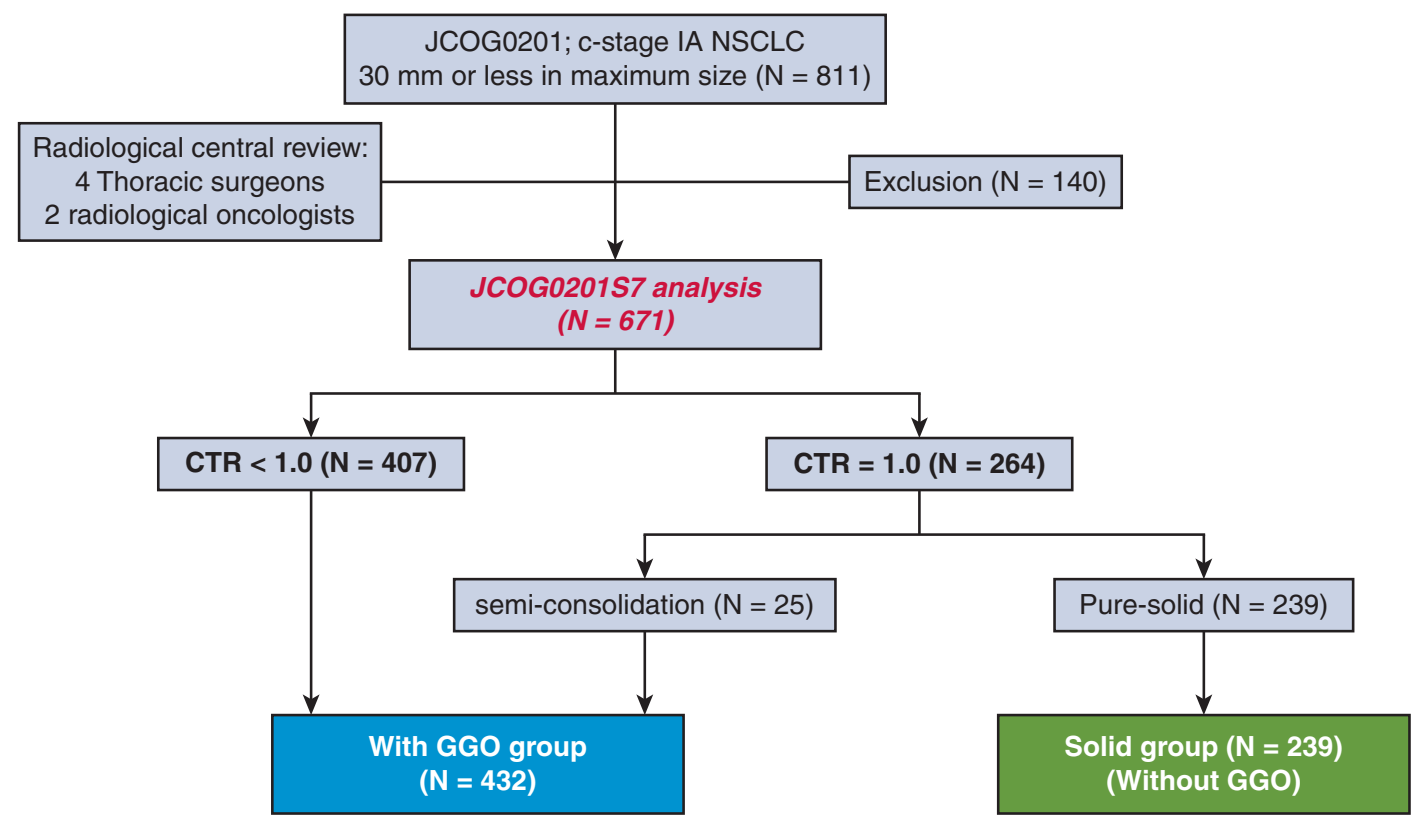

FIGURE 1. Flow chart of patients. Between December 2002 and May 2004, 811 patients were enrolled. Of these, 140 were excluded on the basis of ineligibility criteria including noncancerous lesions. Finally, the prognosis of 671 patients $(82.7 \%)$ was studied on the basis of presence of a GGO component on thin-section CT scan. Of these, $432(64 \%)$ were classified in a with GGO group and $239(36 \%)$ were classified in a solid group according to the result of the radiological central review board. NSCLC, Non-small cell lung cancer; $C T R$, consolidation tumor ratio; GGO, ground-glass opacity.

$\mathrm{c}-\mathrm{T} 1 \mathrm{~b}=85.9 \%, \mathrm{c}-\mathrm{T} 1 \mathrm{c}=73.7 \%$, Figure 4, $B, 5$-year RFS: $\mathrm{c}-\mathrm{T} 1 \mathrm{a}=75.0 \%, \mathrm{c}-\mathrm{T} 1 \mathrm{~b}=75.0 \%, \mathrm{c}-\mathrm{T} 1 \mathrm{c}=58.9 \%)$. Differences in OS based on the presence or absence of a GGO component in each clinical $\mathrm{T}$ category were also significant in the $\mathrm{c}-\mathrm{T} 1 \mathrm{~b}$ and $\mathrm{c}-\mathrm{T} 1 \mathrm{c}$ groups (c-T1a with GGO vs c-T1a solid: HR, 1.41, 95\% CI, 0.19-10.6, c-T1b with GGO vs c-T1b solid: HR, 1.95, 95\% CI, 1.20-3.17, c-T1c with GGO vs c-T1c solid: HR, 2.39, 95\% CI, 1.12-5.09). Figure 5 shows the comparison of survival between the with GGO and solid groups. The survival differences were obvious, and the prognosis of the with GGO group was significantly better than for the c-T1b or c-T1c solid tumors.

Lung cancer recurrence was observed in 102 patients (24 in the with GGO group and 78 in the solid group). The 5 -year cumulative incidence of lung cancer recurrence was $12.8 \%$ overall and significantly different between the with GGO group and solid group $(4.2 \%$ vs $28.5 \%$, Gray's test $P<.0001$ ). Regarding recurrence, 12 local recurrences, 6 distant, and 6 local and distant recurrences were detected in the with GGO group, whereas 37 local recurrences, 26 distant, and 15 local and distant recurrences were detected in the solid group.

\section{DISCUSSION}

On the basis of a previously reported concept concerning the prognostic value of a GGO component and its effect on clinical $\mathrm{T}$ classification in NSCLC staging, ${ }^{9}$ this study aimed to demonstrate the prognostic importance of the presence of a GGO component in early-stage NSCLC using the long-term follow-up data of JCOG0201 in Japan. The strong points of this study are that prospectively collected data with a long-term follow-up period is available for the cohort in Japan, and strict evaluation was conducted on the basis of the radiological central review of thin-section CT scan by experienced thoracic oncologists. As a result, this study reliably validated the fact that the oncological outcomes of early-stage NSCLC differ based on the presence of a GGO component. That is, significant center validation suggested the favorable prognostic impact of the presence of a GGO component in the prospective JCOG0201 dataset. Thus, the presence or absence of a GGO would be considered as an important $\mathrm{T}$ parameter in the next clinical $\mathrm{T}$ classification.

In the 8th edition of the clinical TNM classification, one important proposal was to measure the size of the solid component excluding the GGO particularly in part-solid lung adenocarcinoma to assign a clinical $\mathrm{T}$ factor. ${ }^{8}$ There is no doubt that the size of the solid component better reflects malignant potential than the overall tumor size in lung adenocarcinoma based on several retrospective evidences. ${ }^{4-7}$ However, several debates are arising over the application of radiological solid size to the current 8th edition of TNM classification for the reasons discussed 
TABLE 1. Clinicopathologic characteristics based on a ground-glass opacity presence in clinical stage IA non-small cell lung cancer

\begin{tabular}{|c|c|c|c|}
\hline & With GGO $(n=432)$ & Solid $(n=239)$ & $P$ value* \\
\hline Sex (male) & $153(35)$ & $134(56)$ & $<.0001$ \\
\hline Age (y) (median, range) & $62(36-75)$ & $60(27-75)$ & .3734 \\
\hline \multicolumn{4}{|l|}{ Operative procedures } \\
\hline Pneumonectomy & $0(0)$ & $1(0)$ & .0001 \\
\hline Lobectomy & $344(80)$ & $210(88)$ & \\
\hline Bilobectomy & $5(1)$ & $3(1)$ & \\
\hline Segmentectomy & $35(8)$ & $18(8)$ & \\
\hline Wedge resection & $47(11)$ & $5(2)$ & \\
\hline Exploratory thoracotomy & $1(0)$ & $2(1)$ & \\
\hline \multicolumn{4}{|l|}{ Central review of the CT scan } \\
\hline Maximum tumor size (mm) (median, range) & $20(7-30)$ & $19(8-30)$ & .8385 \\
\hline $0-10(\mathrm{~mm})$ & $30(7)$ & $8(3)$ & \\
\hline $11-20(\mathrm{~mm})$ & $220(51)$ & $136(57)$ & \\
\hline $21-30(\mathrm{~mm})$ & $182(42)$ & $95(40)$ & \\
\hline Solid component size (mm) (median, range) & $11(3-30)$ & $19(8-309)$ & $<.0001$ \\
\hline $0-10(\mathrm{~mm})$ & $215(50)$ & $8(3)$ & \\
\hline $11-20(\mathrm{~mm})$ & $181(42)$ & $136(57)$ & \\
\hline $21-30(\mathrm{~mm})$ & $36(8)$ & $95(40)$ & \\
\hline CTR & $0.57(0-1.0)$ & 1.0 & $<.0001$ \\
\hline Pleural tail (present) & $281(65)$ & $168(70)$ & .1716 \\
\hline Air-bronchogram (present) & $352(81)$ & $81(34)$ & $<.0001$ \\
\hline Margin (hazy/distinct) & $52(12) / 380(88)$ & $3(1) / 236(99)$ & $<.0001$ \\
\hline \multicolumn{4}{|l|}{ Nodal dissection $\dagger$} \\
\hline Hilar nodal dissection or less & $168(39)$ & $59(25)$ & $<.0001$ \\
\hline Mediastinal nodal dissection & $263(61)$ & $179(75)$ & \\
\hline \multicolumn{4}{|l|}{ Pathological diagnosis } \\
\hline Adenocarcinoma & $429(99.3)$ & $221(92)$ & $<.0001$ \\
\hline Squamous cell carcinoma & $2(0.5)$ & $7(3)$ & \\
\hline Large cell carcinoma & $1(0.2)$ & $4(2)$ & \\
\hline Adenosquamous cell carcinoma & $0(0)$ & $3(1)$ & \\
\hline Others & $0(0)$ & $4(2)$ & \\
\hline
\end{tabular}

Differentiation Well/moderate/poor

$379(88) / 39(9) / 5(1)$

88 (37)/93 (39)/38 (16)

Pathological tumor size $(\mathrm{mm})$

$0-10(\mathrm{~mm})$

$11-20(\mathrm{~mm})$

21-30 (mm)

$>30(\mathrm{~mm})$

Size of central fibrotic lesions (mm) $\|$ (median, range)

0-10 (mm)

$16(5-50)$

18 (2-37)

.0003

84 (19)

$239(55)$

22 (9)

98 (23)

130 (54)

77 (32)

9 (2)

7 (3)

5 (0-27)

7 (0-33)

$<.0001$

11-20 (mm)

$344(80)$

$152(64)$

75 (17)

21-30 (mm)

7 (2)

$16(7)$

$>30(\mathrm{~mm})$

0 (0)

1 (0)

Pleural invasion (pl)
pl0
398 (92)

$158(66)$

$<.0001$

$\mathrm{p} 11 / \mathrm{p} 12 / \mathrm{pl} 3$

$24(6) / 6(1) / 0(0)$

$55(23) / 20(8) / 3(1)$

Dissemination\# (present)

Intrapulmonary metastasis** (present)

$2(0)$

4 (2)

.1936

4 (1)

5 (2)

.2924 
TABLE 1. Continued

\begin{tabular}{|c|c|c|c|}
\hline & With GGO $(n=432)$ & Solid $(n=239)$ & $P$ value* \\
\hline \multicolumn{4}{|l|}{ Nodal metastasis (n) $\dagger \dagger$} \\
\hline n0 & $394(91)$ & $193(81)$ & $<.0001$ \\
\hline $\mathrm{n} 1 / \mathrm{n} 2$ & $6(1) / 4(1)$ & $21(9) / 20(8)$ & \\
\hline Vessel invasion $¥$ (positive) & $30(7)$ & $86(36)$ & $<.0001$ \\
\hline Lymphatic invasion $\S \S$ (positive) & $40(9)$ & $88(37)$ & $<.0001$ \\
\hline Additional AAHs (present) & $47(11)$ & $12(5)$ & .0104 \\
\hline
\end{tabular}

Categoric data are shown as numbers (\%) and continuous data as mean \pm standard deviation if normally distributed, and median \pm IQR if not normally distributed (range). Nodal dissection was classified into 2 groups; the hilar node dissection or less and the mediastinal nodal dissection which included both systematic and lobe-specific mediastinal nodal dissection. $G G O$, Ground-glass opacity; $C T$, computed tomography; $C T R$, consolidation tumor ratio; $A A H$, atypical adenomatous hyperplasia. ${ }^{*} P$ value in chi-square test or unpaired $t$ test. †There were 2 missing data. $\ddagger$ There were 7 missing data and 22 unknown findings. §There were 4 missing data and 1 unknown finding. $\|$ There were 12 missing data and 8 unknown findings. There were 4 missing data and 3 unknown findings. \#There were 2 missing data and 1 unknown finding. **There were 4 missing data and 6

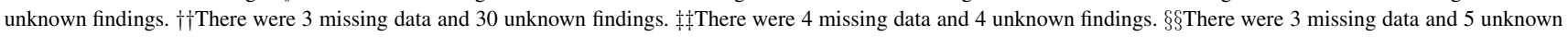
findings.

next. First, there is inconsistency between radiological solid component size and pathological invasive size in part-solid lung adenocarcinomas. ${ }^{13-15}$ One reason for that is the radiological solid area often represents a benign scar or a fibrous scar harboring a stromal invasive component in part-solid tumors pathologically. ${ }^{16,17}$ All the more, there are several findings of part-solid tumors in which the solid component size is difficult or impossible to measure because of the presence of multiple, complicated, or scattered solid areas rather than a single focus. In the eighth edition of the clinical $\mathrm{T}$ classification, there is no consensus on how to make uniform measurements for such solid components in many part-solid tumors. ${ }^{8}$ These include not only typical part-solid tumors ${ }^{18}$ but also atypical part-solid lesions, such as GGO with scattered consolidations, ${ }^{19}$ GGO with island-shaped consolidations, ${ }^{20,21}$ or GGO mimicking organizing pneumonia. ${ }^{22}$ Furthermore, the way of measuring solid component size in part-solid tumors is

TABLE 2. Cox proportional hazard model for clinical stage IA non-small cell lung cancer

\begin{tabular}{|c|c|c|c|c|}
\hline 2A: Overall survival & Univariable HR (95\% CI) & $P$ value* & Multivariable HR $(95 \%$ CI) & $P$ value* \\
\hline Sex (male) & $1.644(1.173-2.306)$ & .0334 & $1.367(0.958-1.950)$ & .0847 \\
\hline Age (y) & $1.052(1.029-1.075)$ & $<.0001$ & $1.048(1.025-1.070)$ & $<.0001$ \\
\hline Solid component size (mm) & $1.087(1.061-1.113)$ & $<.0001$ & $1.072(1.039-1.106)$ & $<.0001$ \\
\hline GGO component (absence) & $3.201(2.265-4.523)$ & $<.0001$ & $1.730(1.076-2.779)$ & .0236 \\
\hline Pleural tail (presence) & $0.988(0.691-1.413)$ & .9464 & $0.686(0.460-1.022)$ & .0637 \\
\hline Air-bronchogram (presence) & $0.556(0.397-0.779)$ & .0007 & $0.881(0.583-1.333)$ & .5490 \\
\hline $\begin{array}{l}\text { Operative procedures } \\
\text { (sublobar resection) }\end{array}$ & $0.878(0.540-1.427)$ & .5990 & $1.265(0.725-2.207)$ & .4072 \\
\hline $\begin{array}{l}\text { Nodal dissection (mediastinal } \\
\text { dissection) }\end{array}$ & $1.195(0.825-1.731)$ & .3462 & $0.918(0.605-1.395)$ & .6898 \\
\hline 2B: Relapse-free survival & Univariable HR (95\% CI) & $P$ value* & Multivariable HR $(95 \%$ CI) & $P$ value* \\
\hline Sex (male) & $1.808(1.317-2.482)$ & .0003 & $1.443(1.035-2.013)$ & .0305 \\
\hline Age (y) & $1.042(1.021-1.063)$ & $<.0001$ & $1.037(1.017-1.057)$ & .0003 \\
\hline Solid component size $(\mathrm{mm})$ & $1.087(1.063-1.111)$ & $<.0001$ & $1.062(1.032-1.093)$ & $<.0001$ \\
\hline GGO component (absence) & $3.781(2.727-5.243)$ & $<.0001$ & $2.169(1.388-3.991)$ & .0007 \\
\hline Pleural tail (presence) & $1.008(0.721-1.409)$ & .9644 & $0.722(0.500-1.045)$ & .0841 \\
\hline Air-bronchogram (presence) & $0.512(0.374-0.701)$ & $<.0001$ & $0.901(0.615-1.321)$ & .5929 \\
\hline $\begin{array}{l}\text { Operative procedures } \\
\quad \text { (sublobar resection) }\end{array}$ & $0.836(0.527-1.325)$ & .4452 & $1.244(0.736-2.100)$ & .4147 \\
\hline $\begin{array}{l}\text { Nodal dissection (mediastinal } \\
\text { dissection) }\end{array}$ & $1.245(0.879-1.763)$ & .2163 & $0.954(0.648-1.407)$ & .8137 \\
\hline
\end{tabular}

Nodal dissection was classified into 2 groups: the hilar node dissection or less and the mediastinal nodal dissection, which included both systematic and lobe-specific mediastinal nodal dissection. $H R$, Hazard ratio; $C I$, confidence interval; $G G O$, ground-glass opacity. $* P$ value in Cox proportional hazard model. 


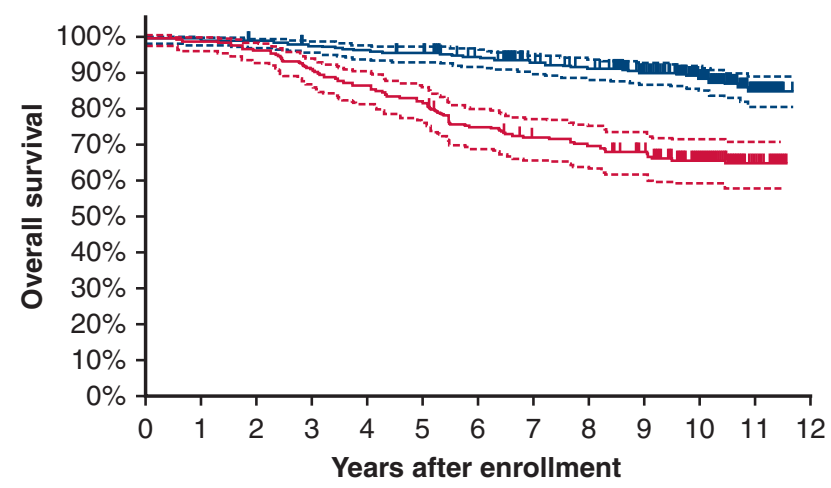

No. at Risk

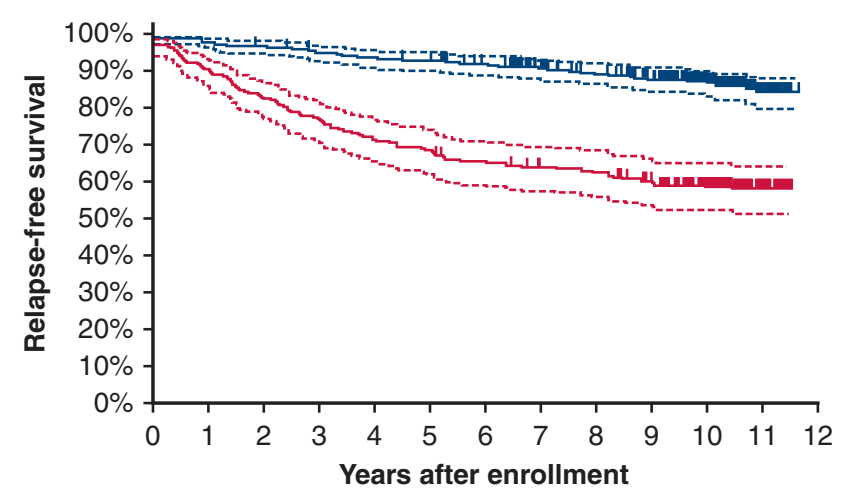

With GGO $432 \quad 427 \quad 424 \quad 418 \quad 412 \quad 408 \quad 396 \quad 368 \quad 353 \quad 334 \quad 261 \quad 67 \quad 0$

Solid $\begin{array}{lllllllllllll}239 & 235 & 228 & 215 & 205 & 193 & 175 & 164 & 159 & 149 & 123 & 37 & 0\end{array}$

A

$$
\text { With GGO Solid }
$$

No. at Risk

With GGO $432 \quad 425 \quad 419 \quad 410 \quad 403 \quad 400 \quad 387 \quad 362 \quad 347 \quad 330 \quad 259 \quad 67 \quad 0$

Solid $\begin{array}{lllllllllllll}239 & 216 & 198 & 185 & 171 & 164 & 155 & 147 & 144 & 133 & 109 & 33 & 0\end{array}$

B

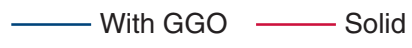

FIGURE 2. Survival outcome based on the presence or absence of a GGO component in clinical stage IA NSCLC (A, OS; B, RFS). The 5-year OS and 5-year RFS were significantly different between the with GGO and solid groups, with a median follow-up time of 10.1 years. GGO, Ground-glass opacity.

available only in lung adenocarcinoma, although several other histological types should be taken into consideration in the clinical $\mathrm{T}$ classification.

Meanwhile, recent institutional reports have noticed a significantly simple fact that the presence of a GGO denotes an influence on the favorable prognosis of NSCLC. More important, our daily practice clearly demonstrates a new issue that radiologic pure-solid lung cancers without a GGO component exhibit a more malignant behavior with regard to both the clinical and pathological aspects, and show several histologic types that have a poorer prognosis than radiologic part-solid lung cancer. ${ }^{9-12,23-28}$ Although this concept has not been fully studied on a nationwide level, a clinical implication of this analysis was that a significant Japanese center validation demonstrated a favorable prognosis of part-solid lung adenocarcinoma in the prospective JCOG0201 dataset, which is irrespective of the solid component size in cases in which the tumor showed a GGO component. This notion is completely different from known information on lung cancer with a part-solid component. Furthermore, the oncological and prognostic differences are immense in early-stage lung

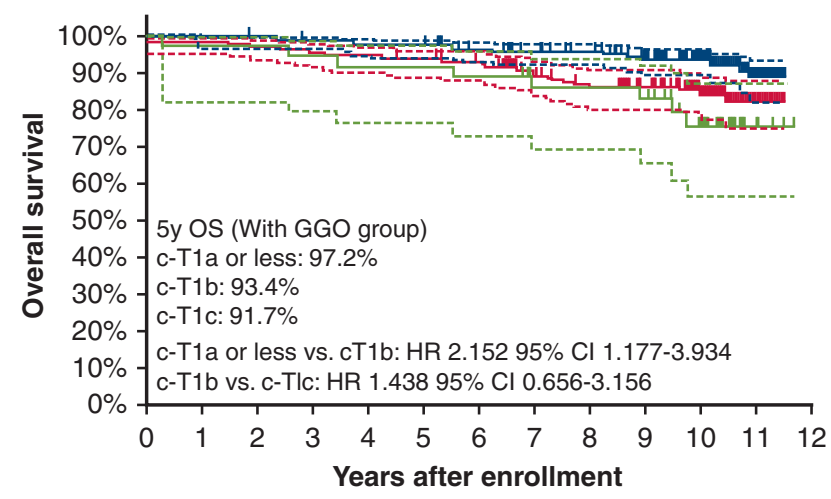

No. at Risk

cT1a or less $215214213211208207200193 \quad 188 \quad 176 \quad 147 \quad 41 \quad 0$

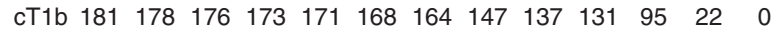
$\begin{array}{llllllllllllll}\text { cT1c } & 36 & 35 & 35 & 34 & 33 & 33 & 32 & 28 & 28 & 27 & 19 & 4 & 0\end{array}$

\section{A}

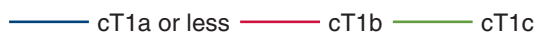

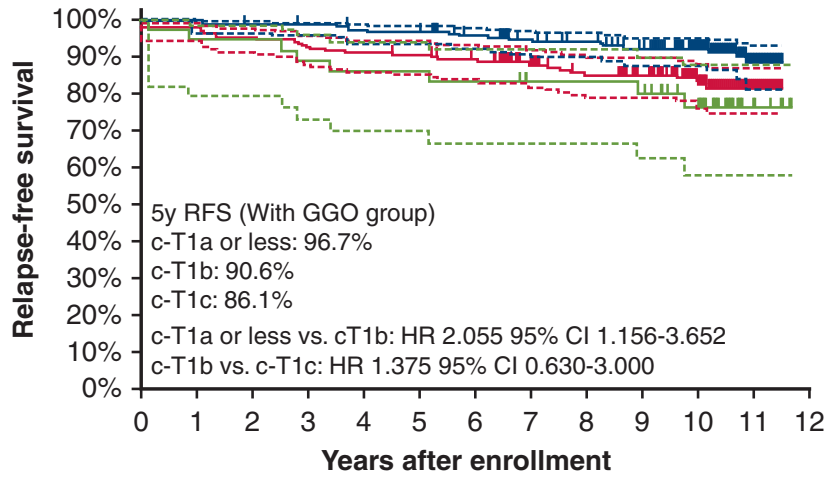

No. at Risk

cT1a or less $215214212210207206199191 \quad 185174 \quad 145 \quad 41 \quad 0$ cT1b $181177 \begin{array}{lllllllllllll}173 & 168 & 165 & 163 & 158 & 144 & 135 & 130 & 95 & 22 & 0\end{array}$

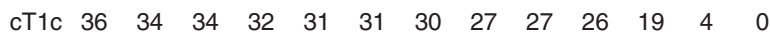

B

FIGURE 3. Survival outcome of the with GGO group in clinical stage IA NSCLC (A, OS B, RFS). The 5-year OS of the with GGO group was excellent, showing $90 \%$ or more of the OS regardless of solid component size. OS, Overall survival; GGO, ground-glass opacity; $H R$, hazard ratio; $C I$, confidence interval; RFS, relapse-free survival. 


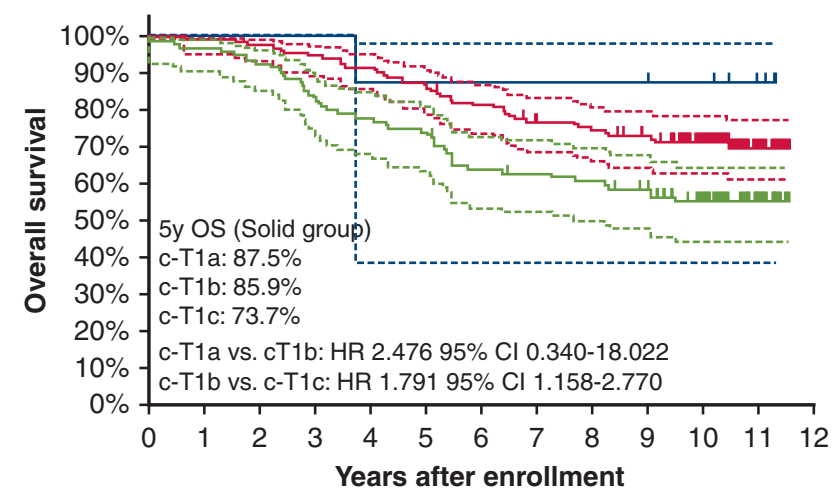

No. at Risk

$\begin{array}{llllllllllllll}\text { CT1a } & 8 & 8 & 8 & 8 & 7 & 7 & 7 & 7 & 7 & 7 & 6 & 3 & 0\end{array}$

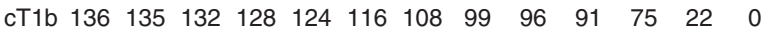

$\begin{array}{llllllllllllll}\text { cT1C } & 95 & 92 & 88 & 79 & 74 & 70 & 60 & 58 & 56 & 51 & 42 & 12 & 0\end{array}$

A

$$
\text { cT1a } \mathrm{cT} 1 \mathrm{~b}-\mathrm{cT} 1 \mathrm{c}
$$

FIGURE 4. Survival outcome of the solid group in clinical stage IA NSCLC (A, OS; B, RFS). Compared with the with GGO group, the prognostic impact of the tumor size was definitive in the solid group, and the survival curve was almost fairly split among the different solid component sizes. OS, Overall survival; $H R$, hazard ratio; $C I$, confidence interval; $R F S$, relapse-free survival.

cancer based on the presence or absence of a GGO as supported in this study. ${ }^{12}$ Especially in lung adenocarcinomas, the significant differences in clinicopathologic and oncologic outcomes between pure-solid and part-solid tumors may be related to their distinctive carcinogenesis., ${ }^{9,12,24,29}$ Obvious distinctions regarding the several baseline characteristics between the with GGO group and solid group is a fundamental biological feature of early-stage lung cancer, which would result in a big difference in prognosis, modes

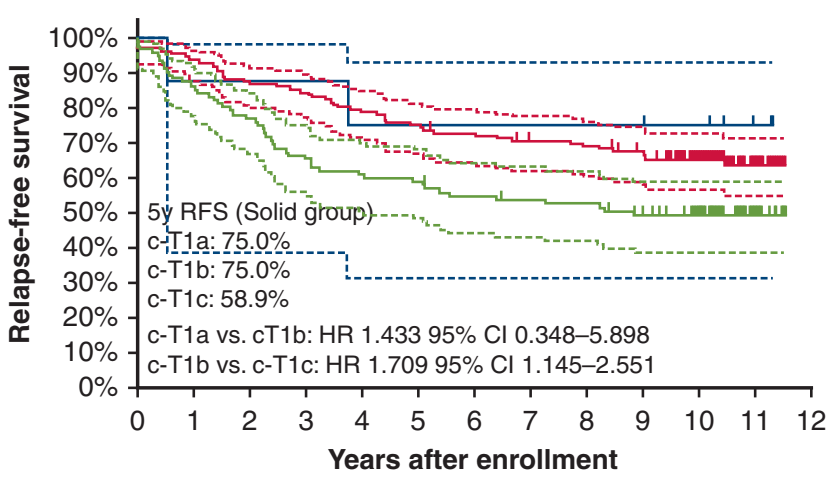

No. at Risk

$\begin{array}{cccccccccccccc}\text { cT1a } & 8 & 7 & 7 & 7 & 6 & 6 & 6 & 6 & 6 & 6 & 5 & 2 & 0 \\ \text { cT1b } & 136 & 127 & 118 & 115 & 107 & 102 & 98 & 92 & 90 & 85 & 68 & 21 & 0 \\ \text { CT1c } & 95 & 82 & 73 & 63 & 58 & 56 & 51 & 49 & 48 & 42 & 36 & 10 & 0\end{array}$




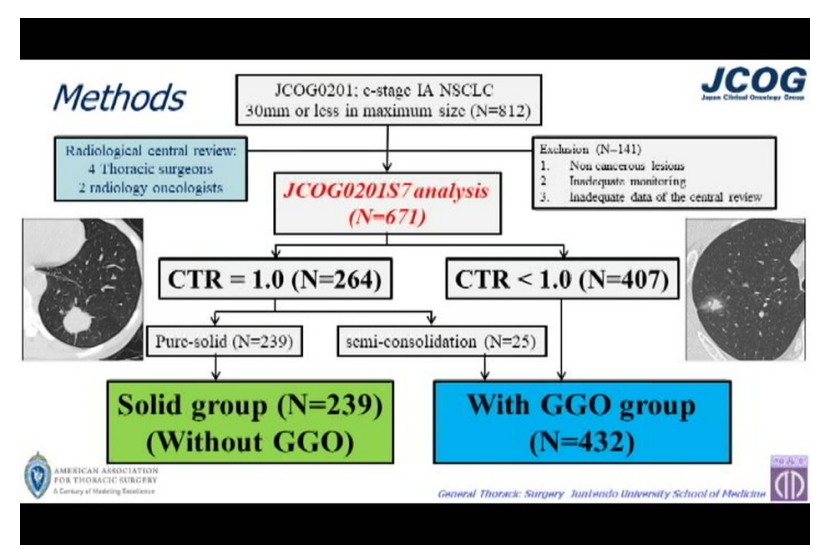

VIDEO 1. The video was presented at the 99th Annual Meeting of The American Association for Thoracic Surgery in Toronto, Canada, 2019. Video available at: https://www.jtcvs.org/article/S0022-5223(20)30734-0/ fulltext.

prognostic for evaluating oncological value, which would be suitable for a universal application. Thus, it would be more reasonable to have a separate staging system for the 2 groups.

When the presence of GGO is recognized as an important $\mathrm{T}$ parameter, one of the discussion points would be the radiologic discrimination between pure-solid NSCLC and solid-dominant part-solid lung adenocarcinoma. However, previous studies rigorously evaluated the clinicopathologic and prognostic differences among them, ${ }^{11,26}$ and this supplemental analysis also supported the finding that presence of even a small GGO component could predict better survival, as shown in the survival result of c-T1c with GGO tumor. In addition to the similar prognostic results between c-T1b and c-T1c with GGO tumor in the current study, the 5-year OS of c-T1c with GGO tumor was excellent, being more than $90 \%$. In this situation, the clinical significance is unclear whether the prognostic category should be subdivided by solid tumor size for such a good prognostic population. Although a superb prognosis of c-T1a or less lesion may require further discrimination in the with GGO group, it is possible to settle the prognosis of part-solid lung cancers with a GGO component as a c-T1a disease based on the survival result of a reliable reference. ${ }^{30}$ In contrast, the survival difference was statistically significant between c-T1b and c-T1c solid tumors, and their survival outcomes behave worse even in the c-stage IA lesion, which would be of value to distinguish them according to tumor size.

With regard to the appropriate operative mode, tumors with a radiologically noninvasive appearance, including cTis and c-T1mi lesions, are candidates for limited surgical resection or close observation. In contrast, major lung resection with mediastinal lymph node dissection is warranted for radiologically invasive lung cancers. The efficacy of intentional segmentectomy is under investigation for small radiologically invasive lung cancers ${ }^{31}$; however, part-solid lung adenocarcinoma with a GGO might be considered a candidate for segmentectomy in the future. In contrast, there were 52 local recurrences in the solid group, which accounted for $22 \%$ of the T1 solid tumors. Lobectomy would be a mainstay for radiologically solid tumors without a GGO component to prevent locoregional recurrence.

\section{Study Limitations}

First, the study data on clinical stage IA NSCLC were limited, and therefore mostly on lung adenocarcinomas showing a tumor size less than $30 \mathrm{~mm}$ in maximum diameter by thin-section CT scan. Hence, the present result cannot be applied to all node-negative lung cancers with a GGO component. However, because most of the lung cancers with a GGO experimentally demonstrate smaller tumor size, ${ }^{9,24}$ we believe that the facts validated in this study are highly valuable to refine the future clinical $\mathrm{T}$ staging. Second, the number of c-T1a solid tumors was small for the analysis. Third, the lack of data on positron emission tomography (PET) would be a limitation of this validation analysis. However, the worldwide application of PET scan is still limited in daily practice. Further studies are needed to elaborate the role of PET in lung cancer staging. Fourth, most of the patients in the JCOG0201 dataset underwent lobectomy, which demonstrated favorable survival outcomes for the with GGO tumor group. However, this does not indicate whether the with GGO group is a proper candidate of sublobar resection. We need to wait for the final results of JCOG0802 in $\mathrm{Japan}^{31}$ regarding the appropriateness of limited surgical resection for such a group. ${ }^{32}$ Finally, the prevalence of early-stage NSCLC with a GGO component seems to be higher in Japan or compared with Europe or North America. We need some sort of validation from other continents with regard to the prognostic value of the presence of a GGO component before advocating a general change in the T-descriptor in the International Association for the Study of Lung Cancer staging system.

\section{CONCLUSIONS}

The favorable prognostic impact of the presence of a GGO component was demonstrated in the JCOG0201 dataset, which was irrespective of the solid component size in cases in which the tumor showed a GGO component (Video 1). Lung cancer with a GGO component is considered as a different oncological category from the solid 
tumor without a GGO. Further studies are required for more refined $\mathrm{T}$ classifications; however, the presence or absence of a GGO should be considered as an important parameter in the next clinical $\mathrm{T}$ classification.

\section{Webcast \\ You can watch a Webcast of this AATS meeting presenta- tion by going to: https://aats.blob.core.windows.net/ media/19\%20AM/Sunday_May5/203BD/203BD/S45\% 20- $\% 20$ Screening $\% 20$ and $\% 20$ early $\% 20$ stage/S45_6_ webcast_083357284.mp4.}

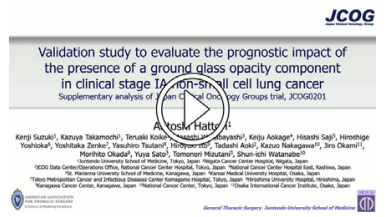

\section{Conflict of Interest Statement}

Dr Suzuki had relevant financial activities outside the submitted work and received research grants and lecture fees from Ethicon, Medtronic, Taiho, and Eli Lilly. Dr Wakabayashi had relevant financial activities outside the submitted work and received lecture fees from Chugai Pharmaceutical Co, Ltd, and Johnson and Johnson K.K. Medical Company. Dr Aokage had the work under consideration for publication and received National Cancer Center Research and Development Fund, Grant-in-Aid for Cancer Research, Practical Research for Innovative Cancer Control Fund, and had relevant financial activities outside the submitted work and received lecture presentation fees from Mochida Pharmaceutical Co, Ltd, and AstraZeneca, seminar presentation fee from MSD. Dr Saji had relevant financial activities outside the submitted work and received grant fees from Taiho, Chugai Pharmaceutical Co, Ltd, Teijin, Eli Lilly, Boehringer Ingelheim, and lecture fees from AstraZeneca, FUJIFILM Medical, Bristol-Myers, Chugai Pharmaceutical Co, Ltd, and Johnson and Johnson K.K. Medical Company. All other authors have nothing to disclose with regard to commercial support.

The authors thank the JCOG Lung Cancer Study Group core members: Yasuhiro Tsutani, MD, Hiroshige Yoshioka, MD, Shiono Satoshi, MD, Hiroyuki Ito, MD, Tadashi Aoki, MD, Kazuo Nakagawa, MD, Jiro Okami, MD, and Morihito Okada, MD, for the conception and interpretation of the article, and Tomonori Mizutani, MD, Ryo Shimoyama, MD, and Haruhiko Fukuda, MD, for the statistical analysis.

\section{References}

1. Suzuki K, Koike T, Asakawa T, Kusumoto M, Asamura H, Nagai K, et al. A prospective radiological study of thin-section computed tomography to predict path- ological noninvasiveness in peripheral clinical IA lung cancer (Japan Clinical Oncology Group 0201). J Thorac Oncol. 2011;6:751-6.

2. Suzuki K, Kusumoto M, Watanabe S, Tsuchiya R, Asamura H. Radiologic classification of small adenocarcinoma of the lung: radiologic-pathologic correlation and its prognostic impact. Ann Thorac Surg. 2006;81:413-9.

3. Asamura H, Hishida T, Suzuki K, Koike T, Nakamura K, Kusumoto M, et al. Radiographically determined noninvasive adenocarcinoma of the lung: survival outcomes of Japan Clinical Oncology Group 0201. J Thorac Cardiovasc Surg. 2013;146:24-30.

4. Maeyashiki T, Suzuki K, Hattori A, Matsunaga T, Takamochi K, Oh S. The size of consolidation on thin-section computed tomography is a better predictor of survival than the maximum tumour dimension in resectable lung cancer. Eur $J$ Cardiothorac Surg. 2012;43:915-8.

5. Tsutani Y, Miyata Y, Mimae T, Kushitani K, Takeshima Y, Yoshimura M, et al. The prognostic role of pathologic invasive component size, excluding lepidic growth, in stage I lung adenocarcinoma. J Thorac Cardiovasc Surg. 2013;146: 580-5.

6. Hwang EJ, Park CM, Ryu Y, Lee SM, Kim YT, Kim YW, et al. Pulmonary adenocarcinomas appearing as part-solid ground-glass nodules: is measuring solid component size a better prognostic indicator? Eur Radiol. 2015;25: 558-67.

7. Burt BM, Leung AN, Yanagawa M, Chen W, Groth SS, Hoang CD, et al. Diameter of solid tumor component alone should be used to establish T stage in lung adenocarcinoma. Ann Surg Oncol. 2015;22(Suppl 3):S1318-23.

8. Travis WD, Asamura H, Bankier AA, Beasley MB, Detterbeck F, Flieder DB, et al. The IASLC Lung Cancer Staging Project: Proposals for Coding T Categories for Subsolid Nodules and Assessment of Tumor Size in Part-solid Tumors in the Forthcoming Eighth Edition of the TNM Classification of Lung Cancer. $J$ Thorac Oncol. 2016;11:1204-23.

9. Hattori A, Matsunaga T, Takamochi K, Oh S, Suzuki K. Prognostic impact of a ground glass opacity component in the clinical T classification of non-small cell lung cancer. J Thorac Cardiovasc Surg. 2017;154:2102-10.

10. Hattori A, Matsunaga T, Takamochi K, Oh S, Suzuki K. Neither maximum tumor size nor solid component size is prognostic in part-solid lung cancer: impact of tumor size should be applied exclusively to solid lung cancer. Ann Thorac Surg. 2016;102:407-15.

11. Hattori A, Matsunaga T, Takamochi K, Oh S, Suzuki K. Importance of ground glass opacity component in clinical stage IA radiologic invasive lung cancer. Ann Thorac Surg. 2017; 104:313-20.

12. Hattori A, Hirayama S, Matsunaga T, Hayashi T, Takamochi K, Oh S, et al. Distinct clinicopathologic characteristics and prognosis based on the presence of ground glass opacity component in clinical stage IA lung adenocarcinoma. J Thorac Oncol. 2019;14:265-75.

13. Thunnissen E, Beasley MB, Borczuk AC, Brambilla E, Chirieac LR, Dacic S, et al. Reproducibility of histopathological subtypes and invasion in pulmonary adenocarcinoma. An international interobserver study. Mod Pathol. 2012;25: 1574-83.

14. Warth A, Stenzinger A, von Brunneck AC, Goeppert B, Cortis J, Petersen I, et al. Interobserver variability in the application of the novel IASLC/ATS/ ERS classification for pulmonary adenocarcinomas. Eur Respir J. 2012;40: 1221-7.

15. Hsu PK, Huang HC, Hsieh CC, Hsu HS, Wu YC, Huang MH, et al. Effect of formalin fixation on tumor size determination in stage I non-small cell lung cancer. Ann Thorac Surg. 2007;84:1825-9.

16. Suzuki K, Watanabe S, Mizusawa J, Moriya Y, Yoshino I, Tsuboi M, et al. Predictors of non-neoplastic lesions in lung tumours showing ground-glass opacity on thin-section computed tomography based on a multi-institutional prospective study. Interact Cardiovasc Thorac Surg. 2015;21:218-23.

17. Yamada N, Kusumoto M, Maeshima A, Suzuki K, Matsuno Y. Correlation of the solid part on high-resolution computed tomography with pathological scar in small lung adenocarcinomas. Jpn J Clin Oncol. 2007;37:913-7.

18. Matsunaga T, Suzuki K, Takamochi K, Oh S. What is the radiological definition of part-solid tumour in lung cancer? Eur J Cardiothorac Surg. 2017;51:242-7.

19. Matsunaga T, Suzuki K. Hattori A, Fukui M, Kitamura Y, Miyasaka T, et al. Lung cancer with scattered consolidation: detection of new independent radiological category of peripheral lung cancer on thin-section computed tomography Interact Cardiovasc Thorac Surg. 2012;16:445-9.

20. Kim H, Goo JM, Suh YJ, Hwang EJ, Park CM, Kim YT. Measurement of multiple solid portions in part-solid nodules for t categorization: evaluation of prog nostic implication. J Thorac Oncol. 2018;13:1864-72. 
21. Nelson DB, Godoy MCB, Benveniste MF, Shewale JB, Spicer JD, Mitchell KG, et al. Clinicoradiographic predictors of aggressive biology in lung cancer with ground glass components. Ann Thorac Surg. 2018;106:235-41.

22. Ichikawa T, Hattori A, Suzuki K, Matsunaga T, Takamochi K, Oh S, et al. Clinicopathological characteristics of lung cancer mimicking organizing pneumonia on computed tomography-a novel radiological entity of pulmonary malignancy. Jpn J Clin Oncol. 2016;46:681-6.

23. Takamochi K, Nagai K, Yoshida J, Suzuki K, Ohde Y, Nishimura M, et al. The role of computed tomographic scanning in diagnosing mediastinal node involvement in non-small cell lung cancer. J Thorac Cardiovasc Surg. 2000;119: 1135-40.

24. Aokage K, Miyoshi T, Ishii G, Kusumoto M, Nomura S, Katsumata S, et al. Influence of ground glass opacity and the corresponding pathological findings on survival in patients with clinical stage I non-small cell lung cancer. J Thorac Oncol. 2018;13:533-42.

25. Berry MF, Gao R, Kunder CA, Backhus L, Khuong A, Kadoch M, et al. Presence of even a small ground-glass component in lung adenocarcinoma predicts better survival. Clin Lung Cancer. 2017;19:e47-51.

26. Ye T, Deng L, Wang S, Xiang J, Zhang Y, Hu H, et al. Lung adenocarcinomas manifesting as radiological part-solid nodules define a special clinical subtype. J Thorac Oncol. 2019;14:617-27.

27. Yip R, Li K, Liu L, Xu D, Tam K, Yankelevitz DF, et al. Controversies on lung cancers manifesting as part-solid nodules. Eur Radiol. 2018;28:747-59.

28. Yip R, Ma T, Flores RM, Yankelevitz DF, Henschke CI. Survival with parenchymal and pleural invasion of non-small cell lung cancers less than $30 \mathrm{~mm} . J$ Thorac Oncol. 2019;14:890-902.

29. Yatabe Y, Borczuk AC, Powell CA. Do all lung adenocarcinomas follow a stepwise progression? Lung Cancer. 2011;74:7-11.

30. Rami-Porta R, Bolejack V, Crowley J, Ball D, Kim J, Lyons G, et al. The IASLC Lung Cancer Staging Project: Proposals for the Revisions of the T Descriptors in the Forthcoming Eighth Edition of the TNM Classification for Lung Cancer. $J$ Thorac Oncol. 2015;10:990-1003.

31. Nakamura K, Saji H, Nakajima R, Okada M, Asamura H, Shibata T, et al. A phase III randomized trial of lobectomy versus limited resection for small-sized peripheral non-small cell lung cancer (JCOG0802/WJOG4607L). Jpn J Clin Oncol. 2011;40:271-4

32. Hattori A, Suzuki K, Matsunaga T, Fukui M, Kitamura Y, Miyasaka Y, et al. Is limited resection appropriate for radiologically "solid" tumors in small lung cancers? Ann Thorac Surg. 2012;94:212-5.

Key Words: ground-glass opacity, pure-solid tumor, prognosis, clinical-T classification

Discussion

\section{Presenter: Dr Aritoshi Hattori}

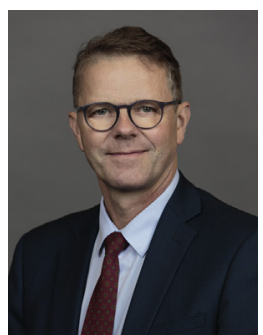

Dr Peter Licht (Odense, Denmark). Although data on GGOs are increasing, the surgical management criteria for these GGOs are not well defined, and we are looking forward to clear surgical guidelines. Dr Hattori and colleagues and other predominantly Japanese research groups have studied and published extensively on GGOs over the last decade. Your previous article showed the prognostic influence of any GGO component in early-stage lung cancer, which was read at the American Association for Thoracic Surgery meeting in Boston 2 years ago and recently published in the Journal. It was considered groundbreaking by many colleagues, and it certainly stirred up some debate because you are suggesting that the $\mathrm{T}$ descriptor of the International Association for the Study of Lung Cancer staging system should not only include the size and location of the tumor but also the behavior of the tumor, meaning that any component of a GGO should influence the T descriptor. It is a radical paradigm shift that you're suggesting. In today's presentation, you aimed to validate these retrospective single-institution retrospective study by using a multicenter prospective cohort.

I have a problem with your results, and I would caution about safely concluding that your earlier findings have now been validated. The baseline characteristics in the 2 groups of the JCOGO201 were significantly different regarding known and important prognostic indicators. Thus, there were substantially more men and more lymph node upstaging in the solid group. Likewise, there was more lymphatic and vessel infiltration in the solid group, and there were more lobar versus sublobar resections in the solid group. Pathology differed substantially, not only the histological subtypes, but also differentiation, which was lower in the solid group. Can we really rely on your conclusion? Please explain why you compared the 2 groups by simple univariate Kaplan-Meier survival analysis and why you did not use a multivariate analysis model such as a Cox proportional hazards model that would have allowed you to adjust for obvious differences in baseline characteristics?

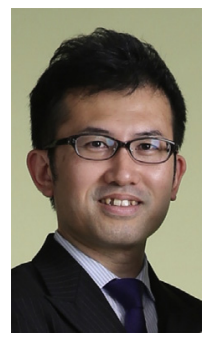

Dr Aritoshi Hattori (Tokyo, Japan). In our previous report published in the Journal, we performed a multivariable analysis including several clinicopathological variables. But in this study, because of the prospective study data, and the study was started in 2002, there are not so many variables to assess by a multivariable analysis. Furthermore, the aim of the study was to validate whether the presence of a GGO component in itself is prognostic or not. So, we did not perform the multivariate analysis and only evaluated the survival outcomes.

Dr Licht. You certainly demonstrated that there's a difference and that GGO has an influence in your univariate analysis. I would have loved to see a multivariate model. My second question relates to the prevalence of early-stage lung cancer with a GGO component, which appears to be significantly more frequent in Japan and perhaps in all of Asia, compared with, for example, Europe or North America. What is your view on validating such data in other 
continents before we consider changing the $\mathrm{T}$ descriptor in the international staging system?

Dr Hattori. Yes. This result is based on the Japanese prospective study data. So, biologically, especially in Asian patients, GGO lung cancer is more common. But this feature is not always prevalent in all the regions. So, if possible, we need a further worldwide validation study.

Dr Licht. Indeed, a word of caution. My last question relates to the use of PET scan in these patients. Previous studies have shown significant differences in SUVmax values between part-solid and solid tumors. You also published on this topic earlier, and yet you decided to use the JCOG0201 cohort in which I believe none had a PET scan. Why would you choose this cohort to validate your previous findings where you did have a PET scan, and what do you think that PET could have added to the data of the JCOG0201?

Dr Hattori. The data with SUVmax are more impressive, but recently, PET scan is not always available all over the world. In contrast, $\mathrm{T}$ descriptor is used worldwide and must be a universal application. So, in that meaning, simply available GGO findings on CT scan is important, but for clinical research, PET scan is more effective for the treatment of lung cancer.

Unidentified Speaker. I just wanted to add that I agree with the findings. In the Journal of Thoracic Oncology, there will be a publication that says the same thing, that they should be divided, there should be a $\mathrm{T}$ descriptor for solid versus part-solid and nonsolid nodules, and we did the Cox regression, the Cox hazard, multivariate analysis, and all of them showed the same thing.

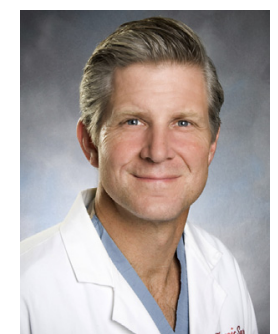

Dr Scott J. Swanson (Boston, Mass). Did you look at the ratio between the amount of GGO in the solid? I could imagine a millimeter of GGO might be different prognostically than 10 or $15 \mathrm{~mm}$, and some people have looked at that before. Did that play into the prognosis, the ratio of the inflammation to the solid piece?

Dr Hattori. With regard to the solid and GGO relation?

Dr Swanson. Right. Of the ones that were part GGO, did it stratify out based on how much GGO relative to the solid component? Did you look at that at all?

Dr Hattori. In the study, we did not evaluate the consolidation to tumor ratio because the most focusing point was the presence or absence of GGO. There are several radiological findings in which it is difficult or impossible to measure the solid component size because of multiple, scattered, or island-shaped solid area compared with those of the single progress. But the most important finding is that regardless of the solid component size, the survival outcome was excellent if the tumor had a GGO component. So, in this study, we did not evaluate the ratio of solid or GGO component. 